Revue d'histoire de l'Amérique française

REVUE D.HISTOIRE DE L'AMÉRIQUE FRANÇAISE

\title{
L’Assemblée législative du Bas-Canada, 1792-1814 : députation et polarisation politique
}

John Hare

Volume 27, numéro 3, décembre 1973

URI : https://id.erudit.org/iderudit/303282ar

DOI : https://doi.org/10.7202/303282ar

Aller au sommaire du numéro

Éditeur(s)

Institut d'histoire de l'Amérique française

\section{ISSN}

0035-2357 (imprimé)

1492-1383 (numérique)

Découvrir la revue

\section{Citer cet article}

Hare, J. (1973). L’Assemblée législative du Bas-Canada, 1792-1814 : députation et polarisation politique. Revue d'histoire de l'Amérique française, 27(3),

361-395. https://doi.org/10.7202/303282ar d'utilisation que vous pouvez consulter en ligne.

https://apropos.erudit.org/fr/usagers/politique-dutilisation/ 


\title{
L'ASSEMBLÉE LÉGISLATIVE DU BAS-CANADA, 1792-1814 :
}

\section{DÉPUTATION ET POLARISATION POLITIQUE}

\author{
JoHN HARE \\ Centre de recherche en \\ civilisation canadienne-française \\ Université d'Ottawa
}

Par l'Acte constitutionnel, le roi acceptait de partager une partie de son autorité avec ses sujets canadiens. C'est ainsi que le Québec, désormais connu sous le nom de Bas-Canada, fit son entrée dans l'ère des révolutions démocratiques et égalitaires. Certes, la souveraineté du peuple serait contestée et ses représentants souvent frustrés dans leurs tentatives d'infléchir la politique du gouvernement. Cependant, si l'apprentissage était difficile, le mouvement était irréversible. Mais comment, en pratique, les Canadiens ont-ils participé à la vie politique à l'intérieur de la Chambre d'Assemblée ? Dans cette étude, nous faisons l'analyse de la députation et de son comportement en Chambre.

Dans une lettre du 21 février 1810, le gouverneur Craig exprimait sa répugnance à côtoyer la plupart des membres de l'Assemblée: "except three or four Englishmen, and a lesser number of Canadians of decent manners, they are people with whom it is impossible I can have any connection not even in the common intercourse of a dinner ${ }^{1 "}$. A ce moment, la Chambre comptait neuf seigneurs, seize avocats et notaires ${ }^{2}$, treize mar-

1 Lettre de Craig à Bunberry, APC, MG, 11, Q. 112: 51-55 (voir Jean-Pierre Wallot, Le Bas-Canada sous l'administration de Craig (18071811), thèse de doctorat présentée à l'Université de Montréal (1965), 339).

2 Les revenus des avocats et des notaires dépassaient généralement la rémunération accordée aux fonctionnaires supérieurs et pouvaient atteindre facilement $£ 350$ par année (voir Gilles Paquet et Jean-Pierre Wallot, "La Liste civile dans le Bas-Canada (1794-1812): un essai d'économie historique", dans RHAF, vol. 24, no 2 (1970): 256-260. On ne doit pas oublier la largesse de certains avocats canadiens à la suite des élections. Jean-Antoine Panet distribua cent louis d'or aux pauvres après son élection en 1792 et en 1800, il promit de déposer cent piastres dans la corbeille de noces de la première mariée de sa circonscription (voir Jean et Marcel Hamelin, Les Mours électorales dans le Québec de 1791 à nos jours (Montréal, Les Editions du Jour, 1962), 32).

[ 361 ]

RHAF, vol. 27, no 3 (décembre 1973) 
chands, un médecin, un agent et un arpenteur. On note aussi cinq cultivateurs dont au moins deux "habitants illettrés": Joseph Meunier et Joseph Duclos. Il est probable que Craig pensait à ces derniers lorsqu'il écrit: "real habitants immersed in ignorance and superstition, without education, manners or the slightest knowledge of business ${ }^{3 "}$.

\section{I - LA DÉPUTATION, 1792-1814}

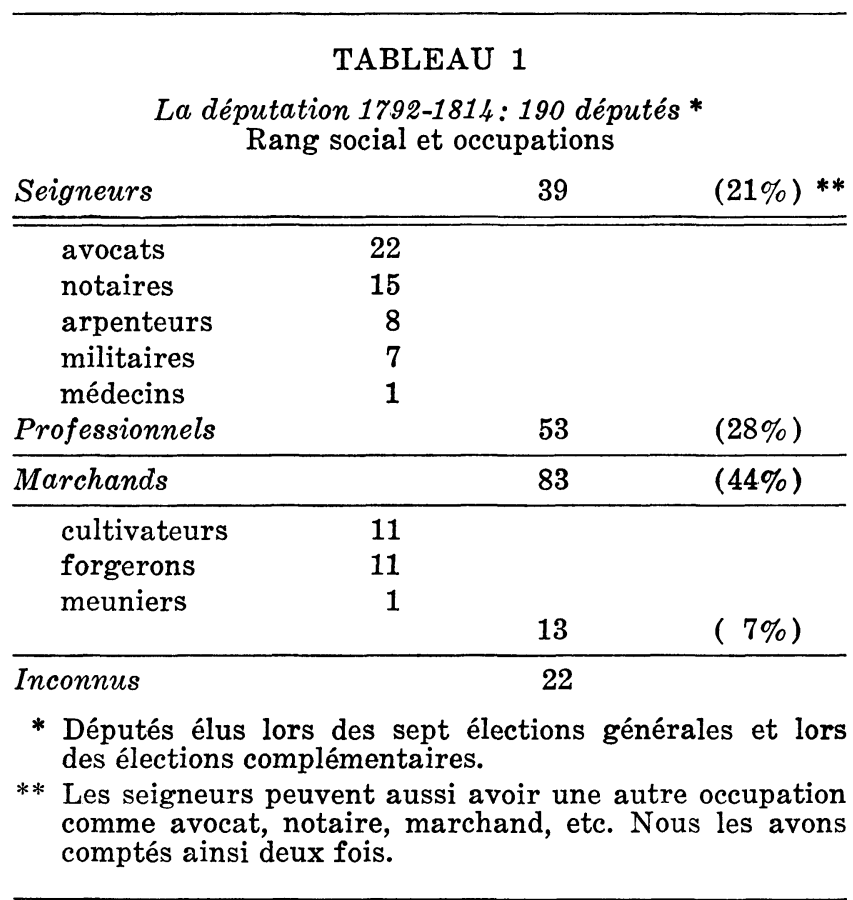

L'examen détaillé de la députation de 1792 à 1814 montre l'importance de deux groupes: les marchands et les professionnels. On constate aussi que les cultivateurs ne constituent qu'une partie peu importante de la députation (voir le tableau des députés à la fin de cette étude).

3 Lettre de Craig, op. cit. Il est intéressant de noter qu'un Canadien, Joseph Godefroy de Tonnancour, député de Buckinghamshire de 1792 à 1796, avait étudié à l'Université d'Oxford, de 1772 à 1774 . 


\section{TABLEAU 2}

La députation 1792-1814

Rang social, occupations et groupes ethniques

\begin{tabular}{|c|c|c|c|c|}
\hline \multirow[b]{2}{*}{ Seigneurs } & \multicolumn{2}{|c|}{ ANGLAIS 52} & \multicolumn{2}{|c|}{ CANADIENS 138} \\
\hline & 11 & $(21 \%)$ & 28 & $(20 \%)$ \\
\hline avocats & 8 & & 14 & \\
\hline notaires & 1 & & 14 & \\
\hline arpenteurs & 2 & & 6 & \\
\hline militaires & 4 & & 3 & \\
\hline médecins & - & & 1 & \\
\hline Professionnels & 15 & $(29 \%)$ & 38 & $(27 \%)$ \\
\hline Marchands & 36 & $(69 \%)$ & 37 & $(26 \%)$ \\
\hline cultivateurs & - & & 11 & \\
\hline forgerons & - & & 1 & \\
\hline meuniers & - & & 1 & \\
\hline & - & & 13 & $(9 \%)$ \\
\hline
\end{tabular}

La grande différence entre la députation anglaise et canadienne réside dans l'importance de l'élément commercial anglais. Par contre, il ne semble y avoir aucun cultivateur dans le groupe britannique. Malheureusement les précisions manquent pour une trentaine de députés canadiens. Une conclusion s'impose cependant: les députés viennent en général des rangs les plus élevés de la société bas-canadienne.

\section{TABLEAU 3}

La députation 1792-1814: Tableau des âges *

\begin{tabular}{|c|c|c|c|c|c|c|c|c|c|}
\hline \multirow[b]{2}{*}{ Elections } & \multirow[b]{2}{*}{$\begin{array}{l}\text { moins } \\
\text { de } 30\end{array}$} & \multicolumn{5}{|c|}{ Age } & & \multirow{2}{*}{$\begin{array}{l}\text { Membres } \\
\text { élus dans } \\
\text { plus d'une } \\
\text { circons- } \\
\text { cription }\end{array}$} \\
\hline & & $30-39$ & $40-49$ & $50-59$ & $60-69$ & $70-79$ & $\begin{array}{l}\text { Inco } \\
\text { Ang. }\end{array}$ & $\begin{array}{l}\text { nnu } \\
\text { Can. }\end{array}$ & \\
\hline 1792 & 3 & 15 & 15 & 9 & 1 & & 4 & 1 & 1 \\
\hline 1796 & 8 & 12 & 5 & 8 & 4 & 1 & 5 & 7 & \\
\hline 1800 & 3 & 12 & 10 & 7 & 4 & & 5 & 9 & \\
\hline 1804 & 5 & 9 & 12 & 7 & 3 & & 4 & 10 & \\
\hline 1808 & 2 & 11 & 15 & 7 & & & 6 & 9 & \\
\hline 1809 & 5 & 9 & 13 & 6 & 1 & 1 & 5 & 8 & 2 \\
\hline 1810 & 3 & 7 & 18 & 6 & 1 & & 3 & 12 & \\
\hline
\end{tabular}


S'il n'y avait aucun membre de moins de vingt et un ans, on note cependant de très jeunes députés: Etienne Guy avait vingt-deux ans lors de son élection en 1796; Jean-Thomas Taschereau, vingt et un ans en 1800; Benjamin-Joseph Frobisher, vingt-deux ans en 1804; Louis-Joseph Papineau, vingt-deux ans en 1809. On ne rencontre que deux députés de soixante-dix ans ou plus: Jean-Marie Ducharme avait soixante-treize ans en 1796 et Joseph Beauchamp, soixante-dix en 1809.

TABLEAU 4

La députation 1792-1814: âge moyen

\begin{tabular}{cccc}
\hline \hline Elections & $\begin{array}{c}\text { Ensemble de } \\
\text { la députation }\end{array}$ & Anglais & Canadiens \\
1792 & 42,1 ans & 41,3 & 42,4 \\
1796 & 42,4 & 32,7 & 45,4 \\
1800 & 46,2 & 36,7 & 49,9 \\
1804 & 42,5 & 39,2 & 43,8 \\
1808 & 41,7 & 37,1 & 43,0 \\
1809 & 42,2 & 36,7 & 44,1 \\
1810 & 43,6 & 37,1 & 45,6 \\
\hline $1792-1810$ & 42,9 & 37,3 & 44,8 \\
\hline
\end{tabular}

L'âge moyen de 42,9 ans ne varia que très peu au cours de la période ${ }^{4}$. La différence entre l'âge moyen des membres canadiens et des membres anglais s'explique en partie par le fait que les dates de naissance de plusieurs nous sont inconnues: dixneuf, c'est-à-dire 37\% des députés anglais à comparer à seulement vingt-cinq députés canadiens $(18 \%)$. Par contre, la tendance se maintient tout au cours de la période. Il semble ainsi que la députation canadienne possédait en général plus de maturité que la députation anglaise.

4 A la Chambre des Communes d'Angleterre de 1754 à 1790, l'âge moyen se situait autour de 43 ans (voir Sir Lewis Namier and John Brooke, The House of Commons, 1754-1790 (London, HMSO, 1964), I: 98). 
TABLEAU 5

La députation 1792-1814: âge moyen des nouveaux membres 5

\begin{tabular}{|c|c|c|c|}
\hline Elections & $\begin{array}{l}\text { Ensemble de } \\
\text { la députation }\end{array}$ & Anglais & Canadiens \\
\hline 1796 & 43,2 ans & $28,0^{a}$ & 46,5 \\
\hline 1800 & 39,3 & $28,2^{\text {b }}$ & 43,3 \\
\hline 1804 & 38,6 & 32,5 b & 41,3 \\
\hline 1808 & 35,6 & $36,0^{\mathrm{c}}$ & 35,5 \\
\hline 1809 & 41,1 & $38,7^{d}$ & 42,7 \\
\hline 1810 & 47,3 & - & 47,3 \\
\hline $1796-1810$ & 40,8 & 38,1 & 43,2 \\
\hline \multicolumn{4}{|c|}{ 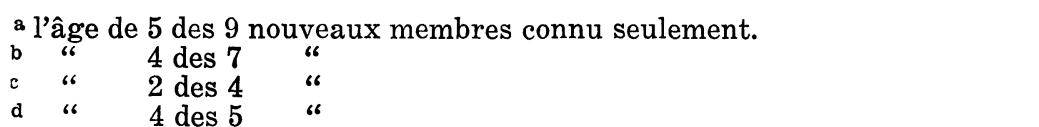 } \\
\hline
\end{tabular}

Les Canadiens constituaient toujours une majorité à la Chambre. De 1792 à 1814, on énumère 190 membres différents dont 138 Canadiens $(72,4 \%)$ et 52 Anglais $(27,4 \%)$. Parmi les "Canadiens", on note un Acadien ${ }^{6}$ et sept Français de naissance ${ }^{7}$; la grande majorité étant née au Québec, soit 94 pour cent. Les “Anglais", par contre, venaient surtout de l'extérieur de la province; seulement cinq nés au Québec, soit 10 pour cent ${ }^{8}$. C'est ainsi qu'au cours des sept élections, on choisit un total théorique de 350 députés, dont 251 Canadiens $(71 \%)$ et 99 Anglais (29\%). Les Canadiens étaient élus surtout dans les circonscriptions rurales, $218(87 \%)$ contre 33 dans les villes $(13 \%)$. La députation anglophone était mieux partagée, 55 dans les comtés ruraux $(56 \%)$ et 44 dans les villes (44\%).

5 En Angleterre, 32,5 ans, ibid.

6 Jean Boudreau, député de Hampshire 1792-1796, né en Acadie en 1747.

7 Jean Digé, né en Normandie, député de Cornwallis de 1792 à 1796;

François Dambourges, né au Béarn, député de Devon de 1792 à 1796; Alexandre Ménut, député de Cornwallis de 1796 à 1804; Philippe de Rocheblave, de la noblesse française, député de Surrey de 1792 à 1802. Si les "Français" de naissance ne représentent que $5 \%$ de la députation de 1792 à 1814, lors du premier parlement de 1792 à 1796, ils représentaient $11 \%$. 8 John Caldwell, né à Québec, député de Dorchester de 1800 à 1813 ; James Cuthbert, né à Berthier, député de Warwick de 1796 à 1811; Ross Cuthbert, né à Montréal, député de Warwick de 1800 à 1816; Benjamin Joseph Frobisher, né à Montréal, député de Montréal de 1804 à 1808; Thomas Lee, né à Québec d'une mère canadienne (il a fait ses études au Séminaire de Québec). Lee fut député de la circonscription de Northumberland de 1809 à 1816. 
TABLEAU 6

Composition ethnique de la députation

\begin{tabular}{ccc}
\hline \hline Elections & Canadiens & Anglais \\
1792 & 35 & 15 \\
1796 & 36 & 14 \\
1800 & 35 & 15 \\
1804 & 34 & 16 \\
1808 & 36 & 14 \\
1809 & 36 & 14 \\
1810 & 39 & 11 \\
\hline
\end{tabular}

Les 21 circonscriptions rurales avaient droit à 39 sièges (tableau 7). Lors des sept élections, les Canadiens firent élire 218 députés $(80 \%)$ et les Britanniques 55 (20\%). Sept circonscriptions ont toujours envoyé des députés canadiens à l'Assemblée: Cornwallis, Orléans, Hertford, Richelieu, Surrey, Kent et Leinster. Seulement Gaspé choisit toujours un député britannique. Bedford n'a élu des Canadiens qu'en 1792 et 1810. Le comté de Warwick envoya dix Anglais contre quatre Canadiens; Saint-Maurice, plus partagé, huit Canadiens contre six Britanniques.

\section{TABLEAU 7}

Circonscriptions: division rurale-urbaine

\begin{tabular}{|c|c|c|}
\hline Circonscription & Sièges & $\%$ \\
\hline 21 rurales & 39 & 78 \\
\hline 4 urbaines & 11 & 22 \\
\hline Total & 50 & \\
\hline
\end{tabular}

A la suite des sept élections, les électeurs des 21 comtés ruraux ont choisi des députés canadiens exclusivement 98 fois $(67 \%)$ et ils ont partagé la députation en 31 occasions $(21 \%)$. En 18 occasions seulement, ont-ils élu une députation anglaise, soit 7 fois dans Gaspé, 5 dans Bedford, 4 dans Warwick et une fois dans Buckinghamshire et dans Saint-Maurice. 


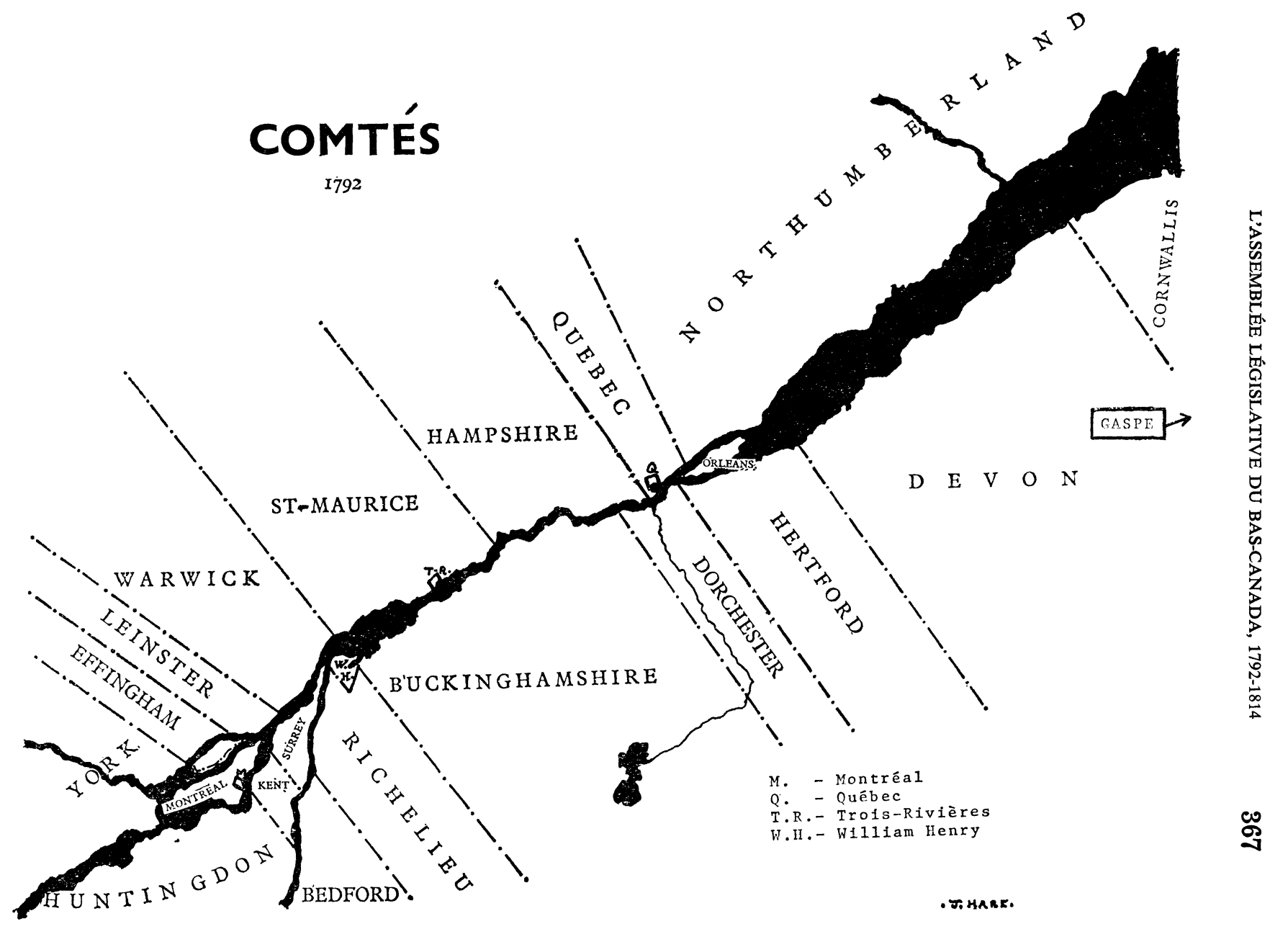




\section{TABLEAU 8}

Composition ethnique de la députation rurale, 179:2-1814

\begin{tabular}{lcc}
\hline \multicolumn{1}{c}{ Circonscription } & Canadiens & Anglais \\
Gaspé (1 député) & - & 7 \\
Cornwallis (2) & 14 & - \\
Devon (2) & 13 & 4 \\
Hertford (2) & 14 & 3 \\
Dorchester (2) & 10 & - \\
Buckinghamshire (2) & 11 & 5 \\
Richelieu (2) & 14 & - \\
Bedford (1) & 2 & - \\
Surrey (2) & 14 & 2 \\
Kent (2) & 14 & 3 \\
Huntingdon (2) & 12 & 3 \\
York (2) & 11 & - \\
Montréal (2) & 11 & 10 \\
Effingham (2) & 11 & 6 \\
Leinster (2) & 14 & 1 \\
Warwick (2) & 4 & 4 \\
Saint-Maurice (2) & 8 & 3 \\
Hampshire (2) & 13 & - \\
Québec (2) & 10 & \\
Northumberland (2) & 11 & 7 \\
Orléans (1) & & \\
\hline
\end{tabular}

Les Anglais ont toujours réussi à conserver une majorité des onze sièges des villes: 44 députés britanniques $(57 \%)$ contre 33 Canadiens $(43 \%)$.

\section{TABLEAU 9}

Composition ethnique de la députation urbaine, 1792-1814

\begin{tabular}{lcc}
\hline \multicolumn{1}{c}{ Circonscription } & Canadiens & Anglais \\
Québec (4) & 13 & 15 \\
Montréal (4) & 14 & 14 \\
Trois-Rivières (2) & 6 & 8 \\
William-Henry (1) & - & 7 \\
\hline
\end{tabular}


TABLEAU 10

Expérience parlementaire

\begin{tabular}{lllllllc}
\hline \hline & 1796 & 1800 & 1804 & 1808 & 1809 & 1810 & $\begin{array}{c}\text { Moyenne } \\
1796-1810\end{array}$ \\
$\begin{array}{l}\text { Nouveaux membres } \\
\text { (élus pour la 1re fois) }\end{array}$ & 37 & 23 & 25 & 19 & 12 & 12 & 21 \\
$\begin{array}{l}\text { Anciens membres } \\
\begin{array}{l}\text { (avec expérience } \\
\text { parlementaire) }\end{array}\end{array}$ & 13 & 27 & 25 & 31 & 38 & 38 & 29 \\
$\begin{array}{l}\text { Pourcentage de } \\
\text { nouveaux }\end{array}$ & $74 \%$ & $36 \%$ & $50 \%$ & $38 \%$ & $24 \%$ & $24 \%$ & $43 \%$ \\
\hline
\end{tabular}

Si les Canadiens conservèrent toujours la majorité en Chambre, néanmoins, les Anglais se trouvèrent surreprésentés puisqu'ils ne comptaient que quinze pour cent de la population. A la suite des élections de 1796, la députation anglaise comptait plus d'expérience parlementaire que la députation canadienne: 40 pour cent des années d'expérience parlementaire contre 28 pour cent de la députation. Aux élections de 1810, les Anglais ne font élire que onze députés (22\% de la députation), et ces députés ne comptent que 20 pour cent des années d'expérience parlementaire. C'est ainsi que la députation canadienne devint plus stable et plus expérimentée.

\section{II - LA POLARISATION POLITIQUE, 1793-1812}

L'analyse des votes en Chambre est rendue difficile par le peu de votes où les noms des votants soient inscrits. En effet, ils ne sont indiqués que si un des membres l'exige. En plus, il y a toujours plusieurs absences pendant les sessions ${ }^{9}$. Malgré les difficultés, nous avons pu analyser les résultats de 147 votes de 1793 à $1812^{10}$.

Une analyse des votes en Chambre vérifie l'hypothèse d'une polarisation des députés, à partir de la première session de 1793. La tendance à voter en bloc se confirme surtout pour le groupe

${ }^{9}$ Les absences oscillèrent autour de $60 \%$ en moyenne jusqu'en 1808. En 1809 et en 1810, les absences tombèrent à $40 \%$ (voir Charles Valois, La Chambre d'Assemblée du Bas-Canada, 1792-1815, thèse de maîtrise présentée à l'Université de Montréal, 1960).

10 Sur les techniques d'analyse, voir Paul G. Cornell, The Alignment of Political Groups in Canada 1841-1867 (Toronto, University of Toronto Press, 1962). 
ministériel (en majorité britannique). En effet, seulement 13,8 pour cent des votes des membres anglais s'expriment contre la majorité du groupe, de 1793 à 1810. Les Canadiens semblent partager leurs votes davantage, soit en moyenne 22,8 pour cent des votes contre le groupe.

TABLEAU 11

Total des votes exprimés par groupe, 1793-1810

\begin{tabular}{lccc}
\hline \hline & $\begin{array}{c}\text { Avec majorité } \\
\text { du groupe }\end{array}$ & $\begin{array}{c}\text { Contre majorité } \\
\text { du groupe }\end{array}$ & Total \\
Ministériels & $856 / 86,2 \%$ & $137 / 13,8 \%$ & 993 \\
Canadiens & $1684 / 77,2 \%$ & $498 / 22,8 \%$ & 2182 \\
TOTAL & $2540 / 80 \%$ & $635 / 20 \%$ & $3175 *$ \\
* 3175 votants sur une possibilité de $6000 ;$ une moyenne de présences de $53 \%$.
\end{tabular}

TABLEAU 12

Votes exprimés selon le groupe ou le "parti"

\begin{tabular}{lcccc}
\hline \hline PARLEMENT & \multicolumn{2}{c}{ MINISTÉRIEL } & & CANADIEN \\
& Avec majorité & Contre & Avec majorité & Contre \\
1er & $90,2 \%$ & $9,8 \%$ & $74,3 \%$ & $25,7 \%$ \\
$2 \mathrm{e}$ & $81,1 \%$ & $18,9 \%$ & $85,9 \%$ & $14,1 \%$ \\
$3 \mathrm{e}$ & $88,2 \%$ & $11,8 \%$ & $67,1 \%$ & $32,9 \%$ \\
$4 \mathrm{e}$ & $84,0 \%$ & $16,0 \%$ & $79,7 \%$ & $20,3 \%$ \\
$5 \mathrm{e}$ & $95,6 \%$ & $4,4 \%$ & $69,9 \%$ & $29,1 \%$ \\
$6 \mathrm{e}$ & $82,6 \%$ & $17,4 \%$ & $85,9 \%$ & $14,1 \%$ \\
\hline moyenne & $86,2 \%$ & $13,8 \%$ & $77,2 \%$ & $22,8 \%$
\end{tabular}

Les historiens considèrent en général que la division en partis politiques n'est évidente qu'à partir de 1805, à la suite de la querelle des prisons et de la parution du Quebec Mercury et du Canadien. Cependant, d'après les statistiques (tableau 12) il semble que la polarisation était plus forte au premier parlement. Dans une lettre à sa femme, Jacques Viger, ancien rédacteur du Canadien, confirme cette tendance à voter en bloc: "Il [le député Jean-Baptiste Durocher] a une cohorte parfaitement 
à sa disposition... qui le suit comme des moutons... et qu'il fait voyager à sa suite dans les divisions avec admiration..." 11 .

\section{a) Le premier parlement, $1793-1796$}

La polarisation des membres devint évidente lors du premier vote du premier parlement sur le choix d'un orateur (tableau 13) et par la suite sur la question des langues (tableau 14). D'autres votes comme ceux sur la restitution du Collège des Jésuites (tableau 15) indiquent que les blocs n'étaient pas irréductibles. Cependant, il semble que, dès la première session, deux blocs se soient constitués, soit un bloc de Canadiens et un bloc ministériel (tableau 16). Bien entendu, il n'est pas possible de partager parfaitement les députés en deux blocs, puisqu'on trouve certains représentants qui votaient à l'occasion avec l'un ou l'autre groupe.

\section{TABLEAU 13}

Premier parlement, première session, 1793:

Division sur le choix de Jean-Antoine Panet comme orateur

Opposés: J. Barnes, T. Coffin, F. Dambourges *, J. Frobisher, W. Grant, J. Jordan, J. Lees, R. Lester, D. Lynd, M. McNider, J. McGill, E. O'Hara, P.-L. Panet*, J. Richardson, L. de Salaberry *, J. Tod, J. Walker, J. Young - 18

(15 - Britanniques, 3 — Canadiens *).

Favorables: P. Bédard, R. Boileau, N. Boisseau, J. Boudreau, B. Cherrier, P.-A. DeBonne, J. Digé, A. Duchesnay, T. Dufour, L. Dunière, G. Dupré, J.-B. Durocher, P. Guerout, J. Lacroix, P. Lavalterie, P. Legras-Pierreville, M. de Lotbinière, F. Malhiot, P. Marcoux, B. Panet, J. Papineau, A. Rivard, P. de Rocheblave, J.-B. Rouville, N. Saint-Martin, G.-E. Taschereau, J.-M. Tonnacour -27

$(0-$ Britannique, 27 - Canadiens $)$.

11 Archives du Séminaire de Québec, Saberdache bleue, tome 1: 193, le 23 avril 1809. Sur la carrière de Jean-Baptiste Durocher (1754-1811), député de Montréal-Ouest de 1792 à 1796 et du comté de Montréal de 1808 à 1811, voir Francis-J. Audet et Edouard-F. Surveyer, Les Députés au Premier Parlement du Bas-Canada (1792-1796) (Montréal, Les Editions des Dix, 1946), 143-204. 


\section{TABLEAU 14}

Premier parlement, première session, 1793:

"Que l'anglais sera considéré comme la langue légale"

Favorables : J. Barnes, F. Dambourges *, J. Frobisher, W. Grant, J. Jordan, J. Lees, R. Lester, M. McNider, J. McGill, E. O'Hara, P.-L. Panet *, J. Richardson, J. Young - 13 (11 - Britanniques, 2 - Canadiens $\left.{ }^{*}\right)$.

Oppostés: $\quad$ P. Bédard, R. Boileau, N. Boisseau, J. Boudreau, B. Cherrier, P.-A. DeBonne, J. Digé, A. Duchesnay, T. Dufour, L. Dunière, G. Dupré, J.-B. Durocher, J. Lacroix, P. Lavalterie, P. LegrasPierreville, G. de Lorimier, M. de Lotbinière, F. Malhiot, P. Marcoux, J. Papineau, A. Rivard, P. de Rocheblave, J.-B. Rouville, N. Saint-Martin, G.-E. Taschereau, J.-M. Tonnacour - 26

( 0 - Britannique, 26 - Canadiens $)$.

\section{TABLEAU 15}

Premier parlement, première session, 1793:

"Que le Collège des Jésuites soit restitué à l'éducation de la jeunesse"

Opposés: J. Barnes, T. Coffin, F. Dambourges*, P.-A. DeBonne*, A. Duchesnay *, G. Dupré *, W. Grant, P. Guerout *, J. Lees, R. Lester, M. de Lotbinière*, D. Lynd, M. McNider, G. McBeath, J. McGill, E. O'Hara, J. Richardson, N. SaintMartin *, G.-E. Taschereau*, J. Tod, J. Walker, J. Young $-22$

(14 - Britanniques, 8 - Canadiens *).

Favorables: P. Bédard, A. Berthelot D'Artigny, R. Boileau, J. Boudreau, B. Cherrier, J. Digé, L. Dunière, J. Lacroix, P. Legras-Pierreville, G. de Lorimier, F. Malhiot, B. Panet, J. Papineau, A. Rivard, P. de Rocheblave, J.-B. Rouville - 17

(0 - Britannique, 17 - Canadiens).

Les membres canadiens appuyant le bloc ministériel avaient des attaches avec l'administration: Pierre-L. Panet, greffier à Québec, François Dambourgès, colonel dans la milice pour la rive sud et Pierre Guérout, juge de paix à Montréal. Parmi les Canadiens "partagés", on note Georges-E. Taschereau, grandvoyer de la région de Québec en 1794, Pierre-A. DeBonne et Antoine Duchesnay, tous deux nommés conseillers exécutifs en 1794 après la première session. Ainsi à partir de la deuxième session, à l'automne de 1794, le spectacle des Canadiens, mem- 
bres du Conseil exécutif, votant contre la majorité canadienne en Chambre, devient familier ${ }^{12}$.

TABLEAU 16

Premier parlement 1793-1796: Tableau général des groupes (18 votes)

BLOC MINISTÉRIEL

J. Lees $15 / 0$ *

R. Lester $16 / 1$

M. McNider $13 / 1$

J. McGill 12/1

E. O'Hara $13 / 2$

J. Richardson 15/3

J. Young 12/1

J. Tod $11 / 0$

J. Barnes 10/0

D. Lynd $10 / 1$

G. McBeath $8 / 0$

T. Coffin $8 / 2$

W. Grant 13/5

\section{BLOC CANADIEN}

P. Bédard 12/1 *

A. Berthelot d'Artigny 12/2

L. Dunière 13/1

$\mathrm{P}$. de Rocheblave $13 / 2$

J.-A. Panet $11 / 0^{b}$

B. Cherrier $10 / 0$ a

A. Rivard $8 / 0^{a}$

J. Boudreau $7 / 0$

P. Marcoux 8/1

J. Papineau $8 / 1$ a

J.-B. Rouville $7 / 1$ a

P. Legras-Pierreville 9/2

Canadiens appuyant le bloc

F. Dambourges $9 / 2$

P. Guerout $5 / 1$ a

P.-L. Panet $6 / 2-3$

G.-E. Taschereau $8 / 6$

Canadiens partagés

P.-A. DeBonne 8/6

A. Duchesnay $7 / 5$

F. Malhiot $6 / 5$

M. de Lotbinière $4 / 3$ b

J.-B. Durocher $3 / 2$ a

J. Digé $7 / 3$

L. De Salaberry $6 / 2$

Membres qui ont exprimé peu de votes

J. Frobisher $4 / 0$

J. Walker $4 / 1$

J. Jordan $3 / 0$

* - le premier chiffre indique le nombre de votes avec le bloc, le deuxième les votes contre.

a - aucun vote aux $3 \mathrm{e}$ et $4 \mathrm{e}$ sessions.

b - orateurs : 1793 - J.-A. Panet; 1794-96 - M. de Lotbinière.
B. Panet $5 / 1$

J.-M. Tonnancour $5 / 1$

R. Boileau $5 / 2$ a

G. Dupré $5 / 3$ a

G. de Lorimier $4 / 0$ a

T. Dufour $4 / 1$ a

N. Saint-Martin $4 / 2$ a

N. Boisseau $3 / 0^{a}$

12 Voici comment un écrivain anonyme décrit cette situation trente ans plus tard: "Dans la première session du premier parlement, après l'établissement de notre présente constitution, tous les membres qui après sont devenus les membres ministériels, ont soutenu une doctrine opposée au système actuel de gouvernement." Le Canadien (7 août 1822), 230. 
En plus d' "acheter" le vote des Canadiens par des postes, l'Exécutif commence à s'occuper de l'origine ethnique des représentants dès 1793. Le 3 janvier 1793, James Monk, solliciteur général, écrit à Sir Evan Nepean, sous-secrétaire d'Etat, qu'il espérait faire élire un autre Anglais à une élection complémentaire dans le comté de Leinster. Son candidat, Georges McBeath fut élu et il appuyait le bloc ministériel.

Lors des élections de 1792, on mettait les électeurs en garde contre les tentatives des marchands britanniques d'obtenir le contrôle de l'Assemblée. Et on proposait une liste de candidats favorables aux intérêts des Canadiens: Antoine Juchereau-Duchesnay et Louis de Salaberry dans le comté de Québec, John Lees et Jean Baillargé dans la Basse-Ville, Georges-E. Taschereau et Berthelot D'Artigny dans la Haute-Ville de Québec. Cette liste proposée par DeBonne devient suspecte lorsque l'on examine le comportement de certains de ces "candidats" une fois élus.

\section{b) Le deuxième parlement, 1797-1800}

A l'ouverture de la première session du deuxième parlement en 1797, on comptait seulement 13 députés du premier parlement, un renouvellement de 74 pour cent de la députation, le plus fort de la période. Par la suite, le renouvellement ne dépasserait pas les 50 pour cent et tomberait même à 22 pour cent en 1810. Pendant les quatre sessions, la tendance à voter en bloc serait un peu moins marquée qu'entre 1793 et 1796, surtout pour le groupe ministériel $(81,1 \%)$. Par contre, le bloc canadien montrerait une plus grande homogénéité: 85,9 pour cent des votes avec le groupe contre seulement 74,3 pendant le premier parlement.

Lors du vote sur le choix de l'orateur, trois Britanniques appuyèrent le groupe canadien: George Allsopp, John Black et William Grant. Le comportement des deux derniers peut surprendre puisque John Black se considère comme le chef du bloc ministériel en Chambre et William Grant ne voterait que quatre fois avec le groupe canadien au cours des 21 votes enregistrés auxquels il participerait. Par contre, les trois Canadiens qui votèrent contre Jean-Antoine Panet, soit Pierre-Amable DeBonne, Louis Foucher et Georges De Lanaudière, tous les trois fonctionnaires, surprennent moins.

Si l'on compare le tableau 17, le tableau général des groupes au deuxième parlement, avec celui du premier parlement (tableau 16), il est frappant de constater l'accroissement du bloc canadien: 
un noyau d'une vingtaine à comparer avec les treize au premier parlement. Par contre, les irréductibles du bloc ministériel diminuent à six en comparaison avec les treize du premier parlement ${ }^{13}$.

TABLEAU 17

Deuxième parlement, 1797-1800: Tableau général des groupes (22 votes)

BLOC MINISTÉRIEL

J. Young $20 / 1$

A. Auldjo $14 / 1$

J. Cuthbert $13 / 0$

J. Lees $18 / 3$

W. Grant 17/4

J. Sewell $7 / 1$

J. Fisher 14/6

$-7$

Canadiens appuyant le bloc

L. Foucher $7 / 2$

G. De Lanaudière $7 / 2^{c}-2$

Britanniques partagés

J. Craigie 11/7

T. Coffin 6/4

G. Allsopp 7/4

J. Black $9 / 4$

$-4$

Canadiens partagés

P.-A. DeBonne 9/8

\section{Bloc CANADIEN}

J. Planté $22 / 0$

F. Huot $20 / 1$

C. Bégin 18/0

D. Durocher $16 / 0$

L. Paquet $16 / 0$

P. de Rocheblave 15/1

P. Bédard 14/0

N. Dorion $14 / 1$

A. Lafontaine $13 / 0^{b}$

E. Guy $12 / 0^{b}$

D. Viger $12 / 0^{b}$

A. Menut $15 / 3$

J. Papineau 12/1

P. Sirois $11 / 0$ a

J. Hetier $11 / 0$ a

B. Cherrier $11 / 0$ a

J. Martineau $13 / 2$

J. Viger $9 / 0$ a

C. Millette $9 / 1$ a

F. Têtu 11/1

A. Dumas $14 / 5$

A. Raby $12 / 4$

L. Dunière $10 / 3$

24 -J. Périneault $8 / 3^{c}$

Membres qui ont exprimé peu de votes

J. Jordan $3 / 0$ a

N. Montour $3 / 1$

B. Panet $6 / 0^{c}$

J. Lacroix $4 / 0$ a

E. O'Hara $2 / 0^{\text {a }}$

a - première session seulement.

b - première et deuxième sessions.

c - première et quatrième sessions.

13 Louis-René de Léry écrit dans une lettre du 19 avril 1798: "Il y a une opposition de MM. Young, Grant, Lees et Cuthbert. Cette minorité est faible et, comme dit Mr. Young, in point of number. Young mène la majorité haut la main. Il finissait hier son discours, que des vociférations répétées à l'ordre avaient cent fois interrompu. Chassez-moi, s'écria-t-il, je le désire, il y a si peu de gloire et d'honneur à siéger parmi such set of men! La majorité est réellement despote." Voir BRH, 32, n 5 (1926) : 288-289. 


\section{c) Le troisième parlement, 1801-1804}

Lors du troisième parlement, le groupe canadien s'effrite. On ne compte que 67,1 pour cent des votes avec le groupe, tandis que le bloc ministériel s'affirme: 88,2 pour cent des votes avec le groupe. Le tableau général des groupes (tableau 18) confirme ces tendances. L'Exécutif semble en voie de briser la résistance des Canadiens.

TABLEAU 18

Troisième parlement 1801-1804: Tableau général des groupes (26 votes)

BLOC MINISTÉRIEI

J. Craigie 23/1

J. Steele 19/1

J. Young 16/2

R. Lester $15 / 0$

J. Lees $15 / 1$

J. McGill 15/1

M. Bell $14 / 2$

T. Coffin 11/4

J. Caldwell 10/1

J. Cuthbert $9 / 0$

J. Sewell $8 / 0$

T. Walker $8 / 0$

F. Badgely $6 / 0$ a

A. Shaw $5 / 0$ a

R. Cuthbert $5 / 1^{\mathrm{b}}-15$

Canadiens appuyant le bloc

A. Menut 14/4

P.-L. Panet 12/1

L. Foucher $10 / 2$

P.-A. DeBonne 16/7

A. Raby $12 / 7$

J.-B. Raimond 5/3
BLOC CANADIEN

A. Berthelot d'Artigny 23/0

M. Tellier $23 / 2$

J. Planté 18/2

P. Bédard 16/2

J.-F. Perrault 17/4

A. Menard Lafontaine $14 / 2$

L. Blais $11 / 0$

F. Huot $17 / 6$

J. Boucher 13/4

L. Paquet $11 / 2$

F. Bernier $9 / 1$

J. Archambault $7 / 0$ b

J. Bedard $7 / 0^{\mathrm{b}}$

14 -J.-M. Poulin 13/6

Non-Canadiens appuyant le bloc

1 -G. Vondenvelden $10 / 2$ a - une session.

b - deux sessions.
Canadiens partagés

J. Martineau 12/7

L. Hubert $4 / 4$

A. Caron $6 / 2$

B. Peltier $4 / 0^{b}$

F. Vigé $9 / 5$

$\mathrm{T}$. Taschereau $12 / 10$

Membres qui ont exprimé peu de votes

J. Beaumont $1 / 0$ a

L. Gouin $7 / 4$

F. Lévesque $6 / 5$

B. Livernois $3 / 1$ a

A. Nadon $2 / 0$ a

J. Papineau $1 / 1$ a

$P$. de Rocheblave $6 / 3$ 
La question de l'expulsion de Charles Bouc reviendra en 1801. Charles Bouc, organisateur du groupe canadien dans la région de Montréal, s'attira les foudres de l'Exécutif. On le fit expulser en 1800 à cause d'une prétendue condamnation pour fraude. Lors de la quatrième session du deuxième parlement, la Chambre se divisa sur le principe de le faire entendre avant de l'expulser (tableau 19A). Ensuite, au cours de la première et de la deuxième sessions du troisième parlement, on vota sur l'expulsion de Bouc (tableau 19B). Si les Britanniques ne voulaient même pas le faire entendre, les Canadiens étaient plus partagés sur l'opportunité de le garder en Chambre.

\section{TABLEAU $19 \mathrm{~A}$}

Deuxième parlement, quatrième session, 1800: Division sur le principe de faire entendre Charles Bouc avant de l'expulser

OPposÉs: G. Allsopp, A. Auldjo, F. Bernier*, J. Black, N. Coffin, T. Coffin, J. Craigie, J. Cuthbert, J. Fisher, J. Lees, A. Menut*, J. Sewell, J. Young - 13

(11 - Britanniques, 2 - Canadiens *.)

Favorables: P. Bédard, C. Bégin, M. Berthelot D'Artigny, A. Dumas, D. Durocher, W. Grant**, J. Martineau, J.-A. Panet, J. Papineau, L. Paquet, J. Planté, P. de Rocheblave, P. Taché, F. Huot - 14

(1 Britannique **, 13 - Canadiens.)

TABLEAU 19B

Troisième parlement: Sur l'expulsion de Charles Bouc Première session - 33 membres; Deuxième session — 24 membres

\begin{tabular}{lrll}
\hline \hline OPPOSÉS & & FAVORABLES & \\
1 ère & $2 \mathrm{e}$ & 1 ère & $2 \mathrm{e}$ \\
0 Britannique & 0 Britannique & 13 Britanniques & 7 Britanniques \\
7 Canadiens & 10 Canadiens & 13 Canadiens & 7 Canadiens
\end{tabular}

Trois autres questions semblent avoir cristallisé les oppositions: le projet de loi pour établir des écoles publiques ${ }^{14}, \mathrm{le}$

${ }^{14}$ Ce projet de loi présenté par J.-F. Perrault voulait contrecarrer le projet officiel qui donna naissance aux écoles royales. Voir Jean-Jacques Jolois, J.-F. Perrault (1753-1844) et les origines de l'enseignement laïque au Bas-Canada (Montréal, P.U.M. 1969), 92. 
second, pour rémunérer les membres, et la question de l'absence sans permission de Joseph Papineau. Le seul non-Canadien qui appuyait le bloc canadien lors des trois votes était G. Vondenvelden. Des douze Canadiens qui votèrent contre l'une ou l'autre de ces questions, on remarquait P.-A. DeBonne (opposé aux trois), L. Gouin, A. Menut, T. Taschereau et F. Vigé (opposés à deux), L. Foucher, A. Martineau, J.-M. Poulin, A. Raby et M. Tellier (opposés à une seule). Par contre, J.-M. Poulin et M. Tellier votèrent en faveur du projet sur les écoles publiques; A. Menut, M. Tellier et $F$. Vigé pour la rémunération des membres; A. Martineau, A. Raby et T. Taschereau pour entendre Joseph Papineau.

Les Canadiens qui appuyaient le bloc ministériel (tableau 18) avaient tous des attaches avec l'administration: A. Menut, ancien cuisinier du général Murray, devenu hôtelier; P.-L. Panet et P.-A. DeBonne conseillers exécutifs; L. Foucher, solliciteur général.

\section{d) Le quatrième parlement, 1805-1808}

La vie politique s'envenima à partir de 1805. Au cours du quatrième parlement, on compte 39 votes enregistrés, une augmentation de 50 pour cent sur les années 1801 à 1804 . Le bloc ministériel groupe un noyau de 12 députés (11 Britanniques et P.-A. DeBonne), tandis que le bloc canadien groupe une vingtaine de députés appuyés par un Britannique, John Blackwood, à l'occasion (tableau 20).

Le projet de loi qui divisa l'Assemblée le plus sûrement fut celui sur le financement de la prison de Québec (tableau 21). La majorité canadienne réussit à emporter une série de votes sur la question. Il est probable que l'attitude intransigeante des Britanniques au cours des années 1805-1810 est le résultat en grande partie de cette confrontation ${ }^{15}$.

Deux autres projets de loi sur des questions qui ne seraient réglées que beaucoup plus tard, divisèrent la Chambre: sur les droits féodaux ${ }^{16}$ et sur la rémunération des membres ${ }^{17}$. A la

15 Voir Jean-Pierre Wallot, "La querelle des prisons (Bas-Canada, 1805-1807)", dans RHAF, 14 (1960-1961) : 61-86; 259-276; 395-407; 559-582.

16 Sur le projet de loi qui autorise les seigneurs à composer pour leurs droits féodaux avec leurs censitaires: 10 Britanniques et 3 Canadiens ( $F$. Bernier, E. Dumont et J. Perrault) opposés; 22 Canadiens et 1 Britannique (W. Grant) favorables.

17 Sept Britanniques et cinq Canadiens (M. Caron, E. Dumont, J. Fortin, L. Foucher et F. LeGendre) opposés; 13 Canadiens et aucun Britannique favorables. 
quatrième session, Louis Bourdages proposa un projet de loi sur l'inéligibilité des juges à siéger à l'Assemblée (tableau 22). Cette question allait créer un climat presque révolutionnaire. En effet, les élections générales de 1809 et de 1810 se feront sur cette question. Au quatrième parlement, seul John Blackwood appuya le groupe canadien lors du vote de principe.

\begin{tabular}{|c|c|}
\hline \multicolumn{2}{|c|}{$\begin{array}{c}\text { TABLEAU } 20 \\
\text { Quatrième parlement 1805-1808: Tableau général des groupes (39 votes) }\end{array}$} \\
\hline BLOC MINISTÉRIEL & BLOC CANADIEN \\
\hline $\begin{array}{l}\text { J. Mure } 33 / 3 \\
\text { J. Richardson } 31 / 1 \\
\text { G. Pyke } 29 / 1 \\
\text { W. Moore } 20 / 0 \\
\text { D. Munro } 21 / 1 \\
\text { J. Young } 16 / 0 \\
\text { B. Frobisher } 15 / 0 \\
\text { J. McGill } 15 / 0^{\mathrm{b}} \\
\text { T. Porteous } 14 / 0 \\
\text { J. Sewell } 10 / 1 \\
\text { J. Cuthbert } 8 / 1 \text { b }-11\end{array}$ & $\begin{array}{l}\text { P. Bédard } 35 / 2 \\
\text { J. Planté } 35 / 4 \\
\text { L. Bourdages } 27 / 0 \\
\text { A. Berthelot d'Artigny } 31 / 5 \\
\text { T. Taschereau } 29 / 4 \\
\text { M. Caron } 27 / 3 \\
\text { J. Cartier } 24 / 1 \\
\text { E.-F. Roi } 23 / 1 \\
\text { J. Fortin } 22 / 2 \\
\text { F. LeGendre } 22 / 2 \\
\text { L. Turgeon } 25 / 5 \\
\text { L. de Salaberry } 22 / 4\end{array}$ \\
\hline $\begin{array}{l}\text { Canadiens appuyant le bloc } \\
\text { P.-A. DeBonne } 12 / 1^{\mathrm{b}} \\
\text { L. Foucher } 8 / 3=2\end{array}$ & $\begin{array}{l}\text { A. Martineau } 19 / 5 \\
\text { P. Weilbrenner } 19 / 5 \\
\text { L. Brodeur } 12 / 0^{\mathrm{b}} \\
\text { J.-M. Poulin } 15 / 4 \\
\text { L. Roi Portelance } 18 / 7 \\
\text { J. Archambault } 9 / 0^{\mathrm{a}} \\
\text { L. Proulx } 11 / 2 \\
\text { P. Lussier } 8 / 0 \\
\text { A. Roi } 13 / 5 \\
\text { J. Mondelet } 17 / 9 \\
\text { F. Vigé } 11 / 3 \\
\text { J.-N. Perrault } 11 / 4\end{array}$ \\
\hline $\begin{array}{l}\text { A. Duchesnay } 6 / 4 \\
\text { E. Dumont } 11 / 8\end{array}$ & $\begin{array}{l}\text { s partagés } \\
\text { J.-B. Raimond } 12 / 7 \\
\text { P. de Rocheblave } 7 / 2 \text { a } \\
\text { F. Bernier } 5 / 2 \text { a } \\
\text { L. Chaboillez } 8 / 7 \text { b }\end{array}$ \\
\hline \multicolumn{2}{|c|}{ Membres qui ont exprimé peu de votes } \\
\hline $\begin{array}{l}\text { J. Blackwood } 11 / 6 \\
\text { W. Grant } 7 / 4 \mathrm{a} \\
\text { R. Cuthbert } 4 / 3 \\
\text { J. Caldwell } 6 / 1 \mathrm{~b} \\
\text { A. McKenzie } 5 / 0^{\text {a }} \\
\text { a - une session seulement. } \\
\text { b - deux sessions. }\end{array}$ & G. de Lanaudière $6 / 0^{a}$ \\
\hline
\end{tabular}


TABLEAU 21

Quatrième parlement, première session, 1805: Le projet de loi pour le financement de la prison par des droits sur les importations

Opposés: L. Chaboillez *, J. McGill, D. Munro, J. Mure, T. Porteous, G. Pyke, J. Richardson, J. Young -8

( 7 - Britanniques, 1 - Canadien.)

Favorables: J. Archambault, P. Bédard, A. Berthelot D'Artigny, L. Bourdages, L. Brodeur, M. Caron, J. Cloutier, E. Dumont, W. Grant **, G. de Lanaudière, F. LeGendre, J. Mondelet, J.-N. Perrault, J. Planté, J. Poulin, J.-B. Raimond, P. de Rocheblave, L. Roi Portelance, E.-F. Roi, L. de Salaberry, L. Turgeon, P. Weilbrenner - 22

(1 - Britannique, 21 - Canadiens.)

TABLEAU 22

Quatrième parlement, quatrième session, 1808 : Divisions sur

l'inéligibilité des juges à siéger à l'Assemblée

$1^{\text {er }}$ vote: la permission de présenter le projet; $2^{2 e}$ vote: le projet

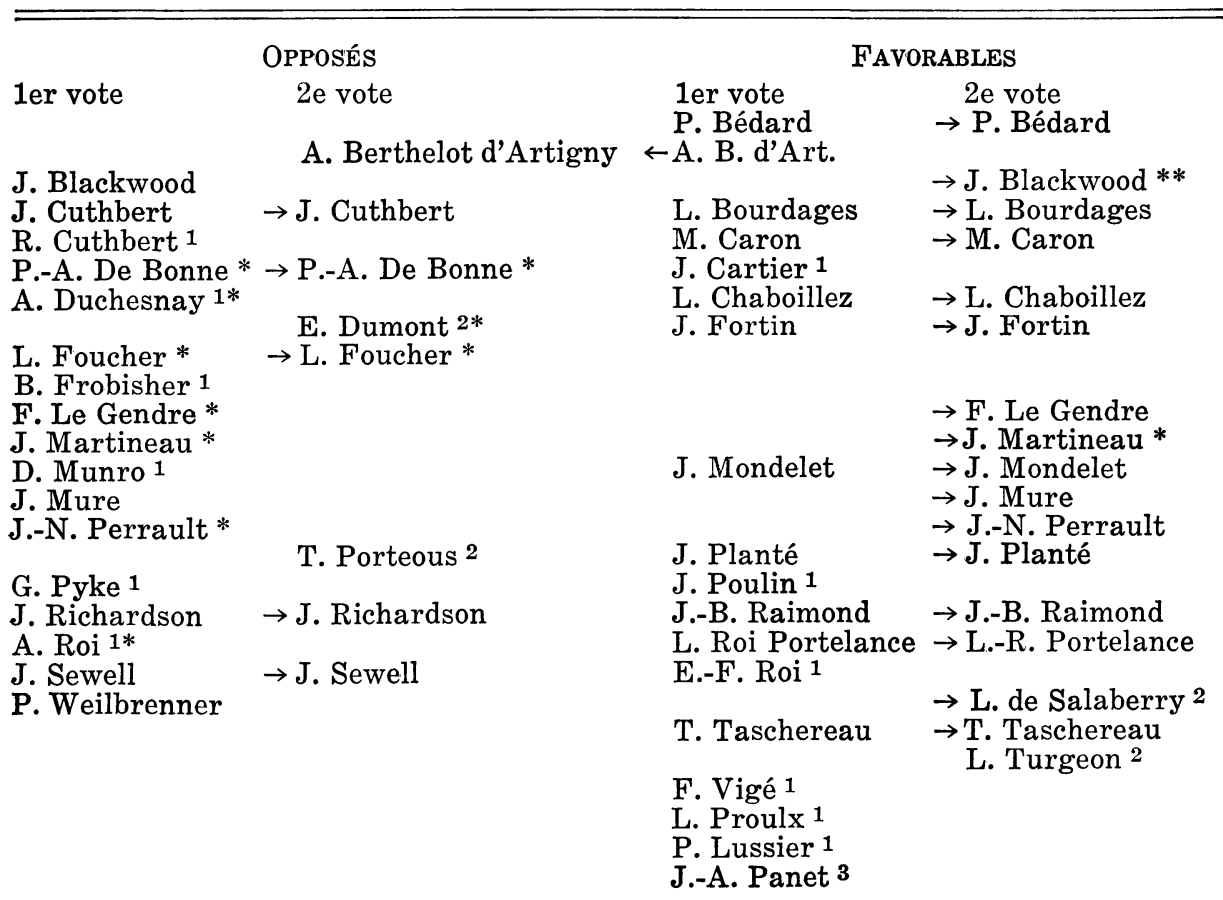

$\begin{array}{ll}9 \text { Britanniques } & 5 \text { Britanniques } \\ 8 \text { Canadiens * } & 3 \text { Canadiens * }\end{array}$

1 premier vote seulement.

2 deuxième vote seulement.

3 L'orateur a voté à cause de l'égalité des voix.
0 Britannique
18 Canadiens
16 Canadiens 


\section{e) Le cinquième parlement, 1809}

En octobre 1809, Le Canadien publia une liste de la députation, indiquant la division en parti ministériel, parti canadien et indépendants (tableau 23). Si l'on compare cette évaluation avec le tableau général des votes enregistrés (tableau 24), on note une grande corrélation entre les deux. Quelques cas cependant méritent discussion:

"Parti ministériel" - J. Faribault et G. Pyke, aucun vote enregistré; J. Mondelet favorisait le bloc canadien quatre fois contre deux.

"Parti canadien" - J. Stuart, aucun vote enregistré; J. Cartier appuyait le bloc ministériel lors du choix de l'orateur; A. Duchesnay vota en faveur de J.-A. Panet mais par la suite appuya le bloc ministériel trois fois (par contre, il vota en faveur de l'exclusion des juges).

"Indépendants" - J. Caldwell et J. Mure appuyaient le bloc ministériel, tandis que J. Jones favorisait le groupe canadien.

\section{TABLEAU 23}

Cinquième parlement, 1809 : Division de la députation en partis selon Le Canadien (octobre 1809)

"PARTi Ministériel": J. Badeaux *, J. Blackwood, T. Coffin, J. Cuthbert, R. Cuthbert, P.-A. DeBonne *, C. Dénéchaud *, J. Faribault*, R. Gray, W. McGillivray, J. Martineau *, J. Mondelet*, W. Moore, J. Planté *, G. Pyke, L. de Salaberry *, J. Turgeon * -17

(8 - Britanniques, 9 - Canadiens *.)

"PARTi CaNAdiEn": P. Bédard, F. Bernier, L. Bourdages, J.-L. Borgia, A. Caron, M. Caron, J. Cartier, H. Delorme, J. Duclos, A. Duchesnay, J.-B. Durocher, J. Fortin, J. Hebert, F. Huot, P. Langlois, P. LeGendre, J. Meunier, J.-A. Panet, L.-J. Papineau, J. Poulin, J. Robitaille, L. Roi, E.-F. Roi, J. Stuart **, D.-B. Viger -25

(1 Britannique **, 24 Canadiens.)

“INDÉPENDANTS": J. Caldwell, J. Jones, J. Mure - 3 
Une seule question agitait la Chambre une fois la motion rituelle du choix de l'orateur votée. En effet, cinq des sept votes de la seule session du cinquième parlement concernaient l'inéligibilité des juges à siéger à l'Assemblée. Malheureusement, le gouverneur Craig prorogea la Chambre avant qu'on ait pu voter sur la question principale. On note 17 favorables, 15 opposés et 9 partagés sur les questions préliminaires (tableau 25). La Chambre fut très divisée en effet; on compte au total 98 votes en faveur et 89 en opposition. Cependant il faut ajouter aux favorables les votes de l'orateur qui pouvait voter en cas d'égalité.

TABLEAU 24

Cinquième parlement, 1809 : Tableau général des groupes (7 votes)

BLOC MINISTÉRIEL

J. Cuthbert 7/0

W. McGillivray $7 / 0$

J. Blackwood $6 / 1$

J. Caldwell $6 / 1$

T. Coffin $6 / 0$

R. Cuthbert $7 / 0$

R. Gray 6/0

J. Mure 6/1

E. Hart 5/0

W. Moore 4/0

J. Sewell $3 / 0-11$

Canadiens appuyant le bloc

P.-A. DeBonne 7/0

C. Dénéchaud $7 / 0$

L. de Salaberry $5 / 0$

J. Planté $5 / 1$

J. Badeaux $5 / 1$

J. Turgeon $4 / 1$

L. Turgeon $5 / 2$

A. Duchesnay $3 / 2$

J. Martineau $3 / 2$

J. Cartier $1 / 0-10$

Aucun vote enregistré

J. Faribault

J. Stuart

\section{Bloc Canadien}

P. Bédard $7 / 0$

L. Bourdages $7 / 0$

J.-L. Borgia $7 / 0$

J.-B. Durocher $7 / 0$

H. Delorme $7 / 0$

J. Hebert $7 / 0$

J. Robitaille $7 / 0$

L. Roi $7 / 0$

J. Trestler $7 / 0$

P. Chagnon $7 / 0$

J. Duclos $6 / 1$

P. Langlois $6 / 1$

J. Meunier 6/1

L.-J. Papineau 6/1

D.-B. Viger $6 / 1$

E.-F. Roi 5/0

A. Caron $4 / 1$

P. LeGendre $4 / 1$

F. Bernier $3 / 0$

M. Caron $4 / 2$

F. Huot $4 / 3$

J. Mondelet 4/2

J. Poulin $4 / 2$

24 -J. Fortin $1 / 0$

Britanniques appuyant le bloc

1 - J. Jones $3 / 2$ 


\section{TABLEAU 25}

Cinquième parlement, 1809 : Cinq votes de procédure sur la question de l'inéligibilité des juges à siéger à l'Assemblée

\section{OPPOSÉS:}

$(5$ votes $5 / 0)$

J. Blackwood

J. Caldwell

T. Coffin

J. Cuthbert

R. Cuthbert

P.-A. DeBonne *

C. Dénéchaud *

R. Gray

W. McGillivray

J. Mure

(4 votes $4 / 0$ )

J. Badeaux *

W. Moore

L. de Salaberry *

(3 votes $3 / 0$ )

E. Hart

J. Sewell - 15

11 - Britanniques

4 - Canadiens *

J. Planté 4/1

J. Turgeon $4 / 1$

L. Turgeon 4/1

A. Duchesnay 3/1

J. Martineau 2/1
Favorables :

(5 votes 5/0)

P. Bédard

L. Bourdages

J.-L. Borgia

H. Delorme

J. Duclos

J.-B. Durocher

J. Hebert

P. Langlois

J. Meunier

L.-J. Papineau

J. Robitaille

L. Roi

J. Trestler

D.-B. Viger

P. Chagnon

(4 votes $4 / 0$ )

E.-F. Roi

(3 votes $3 / 0$ )

17 - P. LeGendre

0 - Britannique

17 - Canadiens

Membres partagés

J. Jones 2/1

M. Caron $3 / 1$

J. Mondelet 3/1

J. Poulin $3 / 1$

\section{f) Le sixième parlement, 1810}

Les élections de 1809 furent très contestées et les deux groupes s'attaquèrent sans merci. On constate la disparition de dixsept anciens députés dont neuf du "parti ministériel", selon Le Canadien (tableau 23): T. Coffin, J. Faribault, W. McGillivray, J. Mondelet, W. Moore, J. Planté, L. de Salaberry, J. Sewell et J. Turgeon. Trois autres appuyaient le bloc ministériel: J. Caldwell, E. Hart et L. Turgeon. Parmi les disparus, seuls P. Chagnon et J. Trestler semblent être sympathiques au bloc canadien quoiqu'ils aient été absents souvent. Les deux autres anciens députés remplacés, soit A. Caron et J. Cartier, participaient peu aux séances de l'Assemblée. 
Le Canadien, en novembre 1809, publia une classification de certains députés du sixième parlement (tableau 26). Le parti ministériel se trouvait affaibli depuis 1809 , ce qui est confirmé par l'analyse du tableau général de la division de la députation (tableau 27).

TABLEAU 26

Sixième parlement, 1810: Division de la députation en partis selon Le Canadien (novembre 1809)

“PARTI MINISTÉRIEL": J. Badeaux *, J. Blackwood, J. Cuthbert, R. Cuthbert, P.-A. DeBonne*, C. Dénéchaud *, R. Gray, J. Martineau * -8 (4-Britanniques, 4 - Canadiens *.)

"PARTI CANADIEN": P. Bédard, F. Bernier, F. Blanchet, L. Bourdages, J. Borgia, M. Caron, H. Delorme, A. Duchesnay, J.-B. Durocher, J. Fortin, J. Hebert, F. Huot, P. Langlois, F. LeGendre, J.-A. Panet, B. Panet, J. Robitaille, E.-F. Roi, J. Stuart**, T. Taschereau, D.-B. Viger - 21

(1 - Britannique **, 20 - Canadiens.)

"INDÉPENDANTS": John Jones, J. Mure - 2

Le caractère démocratique de l'idéologie du parti canadien ressort clairement d'une prise de position sur trois questions fondamentales: les privilèges de l'Assemblée contre toute tentative d'intimidation par l'Exécutif ${ }^{18}$, la nécessité de contrôler les dépenses civiles de la province ${ }^{19}$ et l'inéligibilité des juges à siéger comme député (tableau 28). A chacun des votes, la majorité canadienne opta pour la solution démocratique, tandis que le bloc ministériel s'y opposa.

Aux yeux de Craig, la seule solution fut la dissolution de l'Assemblée et la tenue d'élections. Le 28 février, le Conseil exécutif approuva la publication immédiate d'une proclamation à l'effet de confirmer la fin du parlement provincial et de fixer les dates des nouvelles élections, du 12 mars au 21 avril.

\section{g) Les élections de 1810 et les députés du septième parlement}

Le parti canadien surexcité attaquait les "gens en place" sans merci. Mais le gouvernement était décidé à écraser les Canadiens. Le 17 mars, Craig déposa les trois derniers numéros

18 Huit Britanniques et 3 Canadiens (C. Dénéchaud, A. Duchesnay et L. Gugy) opposés; 22 Canadiens et 2 Britanniques (J. Blackwood et T. Lee) favorables.

19 Sept Britanniques et 2 Canadiens (C. Dénéchaud et L. Gugy) opposés; 20 Canadiens et 1 Britannique (T. Lee) favorables. 


\section{TABLEAU 27}

Sixième parlement, 1810: Tableau général des groupes (8 votes)

BLOC MINISTÉRIEL

M. Bell 8/0

R. Cuthbert $7 / 0$

John Jones $7 / 0$

T. McCord $7 / 0$

J. Mure $7 / 0$

W. Bowen 6/0

J. Blackwood 6/2

R. Gray 5/0

G. Pyke $1 / 0-9$

Canadiens appuyant le bloc

G. Dénéchaud $8 / 0$

L. Gugy $7 / 1-2$

\section{BLOC CANADIEN}

P. Bédard $8 / 0$

F. Blanchet $8 / 0$

J. Beauchamp $8 / 0$

J.-L. Borgia $8 / 0$

M. Caron $8 / 0$

J.-B. Durocher $8 / 0$

B. Panet $8 / 0$

L.-J. Papineau $8 / 0$

F. Bernier $7 / 0$

J. Meunier $7 / 0$

J. Robitaille $7 / 0$

J. Drapeau $7 / 1$

F. Huot $7 / 1$

P. Saint-Julien 7/1

L. Roi $6 / 0$

J. Hebert $6 / 0$

P. Debartzch $6 / 2$

J. Duclos $4 / 0$

D.-B. Viger $4 / 2$

$\mathrm{P}$. Langlois $3 / 0$

E.-F. Roi $3 / 0$

L. Bourdages $2 / 0$

J. Badeaux 2/1

$24-\mathrm{A}$. Duchesnay $1 / 0$

Britanniques appuyant le bloc

1 -T. Lee $7 / 1$

Aucun vote enregistré

J. Cuthbert

P.-A. DeBonne

H. Delorme

F. LeGendre

J. Martineau

J. Papineau

S. Sewell

J. Stuart

du Canadien devant le Conseil exécutif. Après délibération, on signa un avis pour l'arrestation de Charles LeFrançois, l'imprimeur de ce journal. On espérait intimider l'électorat.

Par la suite, le Conseil ordonna la saisie des presses du Canadien et fit emprisonner Pierre Bédard, Thomas 'Taschereau et François Blanchet, les trois principaux rédacteurs et pro- 


\section{TABLEAU 28}

Sixième parlement, 1810: "Que P.-A. DeBonne, étant l'un des juges de la cour du banc du roi, ne peut ni siéger ni voter dans cette chambre"

\begin{tabular}{ll}
\hline \hline OpPosés: & M. Bell, J. Badeaux *, J. Blackwood, E. Bowen, M. Caron *, \\
& J. Cuthbert, R. Cuthbert, P. Debartzch *, C. Dénéchaud *, \\
& A. Duchesnay*, R. Gray, L. Gugy *, John Jones, James Jones, \\
& T. McCord, J. Mure - 16 \\
& (10 Britanniques, 6 - Canadiens *.) \\
FAvorables : & P. Bédard, F. Bernier, F. Blanchet, J. Beauchamp, L. Bour- \\
& dages, J.-L. Borgia, J. Drapeau, J.-B. Durocher, F. Huot, \\
& P. Langlois, T. Lee **, J. Meunier, L.-J. Papineau, B. Panet, \\
& F. Robitaille, L. Roi, T. Taschereau, D.-B. Viger, P. Saint- \\
& Julien - 19 \\
& $(1$ Britannique **, 18 - Canadiens.)
\end{tabular}

priétaires du journal. A la suite de l'arrestation des trois chefs et d'une vingtaine d'autres Canadiens à travers la province parce qu'ils avaient répandu Le Canadien et ses idées, on espérait faire croire à la population qu'il existait un complot révolutionnaire.

Cependant, aux élections, le parti canadien remporta plus de sièges que jamais. Mais la disparition du Canadien et l'emprisonnement de Pierre Bédard calmèrent les esprits. Selon Craig: “... even they [les membres du parti canadien] will be more guarded in their future violence 20 ".

Peu avant son emprisonnement, l'imprimeur Charles LeFrançois publia un feuillet électoral, probablement l'œuvre de l'équipe du Canadien. Nous remarquons encore une fois la terminologie "parti gouvernemental" et "parti canadien" (tableau 29). L'étude du comportement des députés du septième parlement, de 1811 à 1814, sort partiellement des cadres de notre travail qui s'arrête en 1812. Cependant, même un examen superficiel des vingt-sept votes enregistrés lors des sessions de 1811 et de 1812, démontre la justesse de l'aperçu de Craig: les Canadiens se montraient beaucoup moins hardis que lors des sessions de 1808 et de 1809 .

En janvier 1811, Craig analysa les divisions à l'intérieur de la députation. Il voyait, en plus du parti anglais, trois factions parmi les Canadiens: 'there is the appearance of three parties among them. The one, of the most violent of the whole clique, of which Bourdages is the head; another, with the same views, but with much more art, and a cunning that will fre-

${ }^{20}$ Lettre du 24 mars 1810, APC, MG. 11, Q. 112: 56. 
quently deceive themselves, is led by Papineau. The third party affect a sort of nationality; this is Debartzch's party, and is not numérous ${ }^{21}$."

\section{TABLEAU 29}

Septième parlement, 1811: Division de la députation en partis selon un feuillet électoral de 1810

"PARTI GOUVERNEMENTAL" (Membres qui ont voté contre l'exclusion des juges) : M. Bell, E. Bowen, J. Cuthbert, C. Dénéchaud, James Jones, J. Mure -6

"PARTI CANADIEN" (Membres qui ont voté pour l'exclusion des juges): P. Bédard, F. Bernier, F. Blanchet, L. Bourdages, J. Drapeau, J. Durocher, J. Hebert, F. Huot, P. Langlois, T. Lee, J. Meunier, B. Panet, L.-J. Papineau, J. Robitaille, L. Roi, E. Saint-Julien, T. Taschereau, D.-B. Viger -18

"Membres en DOUTE": F. Caron, P. Debartzch - 2

Son analyse correspond-elle à l'évidence des votes enregistrés ? Nous constatons en effet une opposition fondamentale entre certains membres du parti gouvernemental, tels que Thomas Coffin, Claude Dénéchaud et le jeune Louis-Joseph Papineau. Le parti gouvernemental comprend Thomas Coffin, James Irvine, John Mure, George Pyke et Claude Dénéchaud, Edward Bowen et Mathew Bell semblent plus partagés, appuyant le groupe Papineau à l'occasion.

A l'intérieur du groupe canadien, il est difficile de découvrir le "parti" de Louis Bourdages ainsi que celui de PierreDominique Debartzch: Bourdages était absent lors de tous les votes enregistrés sauf trois, et même si Debartzch se trouva en Chambre lors de huit des vingt-sept votes enregistrés, on ne peut déceler aucune similitude entre ses votes et ceux des autres députés. Reste Louis-Joseph Papineau qui vota vingt-sept fois sur vingt-sept! Il s'affirmait déjà à l'Assemblée, même s'il n'avait que vingt-cinq ans en 1811. Parmi les membres qui votèrent au moins quatorze fois comme Papineau, on note François Bellet, Jean-Baptiste Durocher, Pierre Langlois, Denis-Benjamin Viger et Jean-Baptiste Bédard.

La vie politique "paisible" d'avant 1805 masquait une profonde division idéologique à l'intérieur de la colonie. Par la suite, les dissensions éclatent au grand jour à la suite de la

${ }^{21}$ Lettre du 15 janvier 1811, dans Robert Christie, op. cit., 6: 183. 
querelle des prisons et la fondation des journaux de combat: The Quebec Mercury, Le Canadien, Le Courier de Québec et Le Vrai-Canadien. Il est paradoxal de constater que les Canadiens sont devenus les véritables défenseurs des principes du parlementarisme, tandis que l'Exécutif cherchait désespérément à retourner à une forme de gouvernement moins démocratique et moins populaire.

\section{III - LA POLARISATION IDÉOLOGIQUE ET ETHNIQUE}

Fernand Ouellet, dans son Histoire économique et sociale $d u$ Québec, 1760-1850, met en lumière l'importance de la dimension socio-économique des conflits idéologiques. Le professeur Ouellet critique avec raison la "valorisation excessive de nos luttes parlementaires" dans l'historiographie canadienne-française (p. 209). En examinant les attitudes des groupes en présence, Ouellet aurait souhaité plutôt une union de "toutes les couches de la bourgeoisie... afin de réaliser un indispensable ajustement des structures sociales aux structures économiques. Marchands et hommes de professions auraient pu se donner la main en vue d'une réforme globale des institutions de la société traditionnelle" (p. 201).

Cependant, "une telle option supposait la primauté du clivage social sur le clivage culturel et ethnique. Elle impliquait un changement radical de mentalité de la part des professions libérales" (p. 201). Il conclut que sur le plan psychologique la bourgeoisie canadienne n'était pas disposée à accepter ce "défi" (p. 202).

Mais les Britanniques, ces prétendus "capitalistes, épris de renouveau et de progrès économiques” ( $p .200$ ), étaient-ils préparés à respecter la volonté de la majorité du peuple ? Ouellet ne peut pas dissimuler le conservatisme de ces "capitalistes". Pris entre deux formes de "fanatisme" [le mot est de Fernand Ouellet], c'est-à-dire la doctrine politique des hommes de professions "peu conscients des besoins réels de la colonie" (p. 201) et celle de la "clique fanatique, dont Ryland et Mountain étaient les leaders", ils toléraient celle qui les défavorisait le moins.

Cependant, l'identification entre les besoins réels de la colonie et la pensée politique d'une petite minorité de Britanniques 212.

22 "Tensions sociales, naissance du nationalisme, crise politique", 196- 
qui ne désirait que faire fortune à tout prix, fut contestée par le parti canadien:

L'opposition de la bourgeoisie d'affaires à certaines réformes indispensables prenait sa source dans cette crainte de voir les professions libérales imposer, par l'intermédiaire des institutions politiques, leur propre vision de l'avenir du Bas-Canada (p. 201).

$\mathrm{Au}$ fond, ces Britanniques "capitalistes" ne défendaient que leurs propres intérêts.

Au tournant du XIX $\mathrm{XI}^{\mathrm{e}}$ siècle, les Canadiens deviennent conscients des dangers que représentent les menées de certains marchands et fonctionnaires britanniques, avides de gain. En majorité à l'Assemblée, les Canadiens se trouvent exclus de l'administration et de l'élaboration des politiques qui allaient affecter leur avenir. Leur réaction contre cette domination par une petite minorité de conquérants s'inscrit dans le mouvement démocratique et libéral qui continue jusqu'à nos jours à dresser les peuples colonisés contre une minorité, souvent de langue et de culture différentes, qui prétend organiser la vie socio-économique à son seul profit.

La polarisation idéologique entre conservateurs et démocrates se double d'une polarisation selon l'origine ethnique. Le parti ministériel groupait l'ensemble de la députation britannique appuyé par les Canadiens membres du Conseil exécutif, comme Pierre-L. Panet, Pierre-Amable DeBonne et Antoine Juchereau-Duchesnay, ainsi que ceux qui occupaient des postes dans l'administration, comme Louis-Charles Foucher, solliciteur général à partir de 1795 . Or, la minorité britannique contrôlait effectivement l'administration civile de la Province: de 1794 à 1812 , les Anglais occupaient 68 pour cent des postes et recevaient 78 pour cent du montant total du budget ${ }^{23}$. Et ces postes se trouvaient à la disposition de l'Exécutif pour distribution selon son bon vouloir. Il lui était ainsi facile d' "acheter" l'appui de certains Canadiens qui espéraient participer au pouvoir. En

23 Voir Gilles Paquet et Jean-Pierre Wallot, La Liste civile dans le Bas-Canada (1794-1812). Déjà en 1790, Monseigneur Hubert écrit: "On pourrait peut-être ajouter, comme une cause de découragement, la préférence qui $\mathrm{y}$ est donnée pour les charges et emplois publics, aux anciens sujets et même aux étrangers établis dans cette Province sur les Canadiens." De 1792 à 1811, les seuls Canadiens nommés au Conseil législatif font partie de la petite noblesse seigneuriale. Jusqu'en 1803, le Conseil législatif resta fermé aux anciens députés. Voir André Garon, "La Fonction politique et sociale des Chambres hautes canadiennes, 1791-1841", dans Histoire Sociale, $\mathrm{n}^{\circ} 5$ (1970): 66-87. 
effet, l'appât du gain renforçait leur idéologie aristocrato-conservatrice traditionnelle ${ }^{24}$.

Le parti canadien, par contre, constituait une véritable force de réforme dans la colonie. Sans l'appui des Britanniques, ses membres tentaient de faire appliquer la constitution et le parlementarisme britannique. Ils croyaient à la souveraineté du peuple et aux droits de la majorité à participer au gouvernement. Pierre Bédard formulait la doctrine de la responsabilité ministérielle croyant qu'elle était essentielle au bon fonctionnement du gouvernement parlementaire. Les Canadiens voyaient avec amertume une infime minorité s'accrocher aux postes et travailler contre les véritables intérêts de la majorité du peuple.

Le Québec avait fait son entrée à l'ère des révolutions démocratiques et égalitaires, mais le cours de l'évolution serait frustré par la minorité ministérielle (surtout des Britanniques) refusant tout changement qui ne s'accorderait pas avec sa vision du monde.

En 1814, le groupe canadien à la Chambre d'Assemblée rédigea un mémoire à l'adresse du gouvernement britannique où il exposa clairement la situation politique de la colonie. Ce document résume en termes concrets le résultat de vingt ans d'expérience du régime parlementaire:

Nous regardons notre Constitution actuelle comme celle qui est la plus capable de faire notre bonheur, et notre plus grand désir serait d'en pouvoir jouir suivant l'intention de Sa Majesté et de son Parlement; mais malheureusement la manière dont elle a été administrée jusqu'ici lui donne un effet opposé à cette intention ${ }^{25}$.

En effet, le document explique cette situation par "la manière dont se sont formés les partis dans cette province”. En 1791, les "anciens sujets" (c'est-à-dire les Anglais) occupaient la majorité des postes dans le gouvernement; "depuis la Constitution, les choses ont continué sur le même pied" et les fonctionnaires britanniques "sont devenus le parti du Gouvernement". Par ailleurs, les Canadiens composent la majorité à

24 Parmi les 52 députés anglais de 1792 à 1814, il se trouve treize hauts fonctionnaires juges ou conseillers exécutifs. De plus, onze remplissent des fonctions de commissaires ou de juges de paix. C'est ainsi que la moitié avait des fonctions dans l'administration. Les 138 Canadiens sont beaucoup moins heureux: douze hauts fonctionnaires, juges ou conseillers exécutifs et dix-huit postes de commissaires ou de juges de paix, c'est-à-dire seulement $20 \%$ de la députation.

25 Mémoire au soutien de la requête des habitans du Bas-Canada à S.A.R. le Prince Regent [Montréal, s.-d., 1814], 6 p. 
l'Assemblée et "les Anglais avec quelques Canadiens dévoués ont formé la minorité". Or, les conseillers exécutifs ayant été pris dans la minorité, "le Gouvernement n'a aucune communication avec la majorité de l'Assemblée que par le moyen des Conseillers et gens en place de la minorité qui étant rivaux de la majorité sont peu propres à la bien représenter".

La majorité qui ne participe pas aux "plans et projets du Gouvernement" n'a d'autre alternative que "de les passer ou de se trouver en opposition avec la minorité (le Gouvernement)". Par conséquent, les divisions de la Chambre d'Assemblée deviennent "nationales":

Les Anglais d'un côté formant la minorité à laquelle est lié le Gouvernement, et les Canadiens de l'autre formant la majorité à laquelle est attachée la masse du peuple; la chaleur de ces divisions nationales passe de la Chambre d'Assemblée dans le peuple, tout le pays se trouve divisé en deux partis; le parti Anglais du Gouvernement d'un côté, et la masse du Peuple de l'autre.

Les auteurs du Mémoire considèrent cet état de faits comme très dangereux: "L'effet d'une telle administration qui tient continuellement le peuple en opposition avec son Gouvernement ne peut être que bien mauvais."

Ce document constitue une analyse lucide de la situation politique du Bas-Canada. Le problème qu'il soulève ne sera réglé que trente ans plus tard par l'acceptation du principe de la responsabilité ministérielle. Malheureusement, les Canadiens ne détiendront plus alors la majorité à l'Assemblée législative du Canada-Uni. 
LES DÉPUTÉS À L’ASSEMBLÉE LÉGISLATIVE DU BAS-CANADA, 1792-1814

\begin{tabular}{|c|c|c|c|c|c|c|c|}
\hline CIRCONSCRIPTION & $\begin{array}{c}\text { 1er PARLEMENT } \\
1792-1796\end{array}$ & $\begin{array}{c}\text { 2e PARLEMENT } \\
1796-1800\end{array}$ & $\begin{array}{c}\text { 3e PARLEMENT } \\
1800-1804\end{array}$ & $\begin{array}{l}\text { 4e PARLEMENT } \\
1804-1808\end{array}$ & $\begin{array}{c}\text { 5e PARLEMENT } \\
1808\end{array}$ & $\begin{array}{c}\text { 6e PARLEMENT } \\
1809\end{array}$ & $\begin{array}{c}\text { 7e PARLEMENT } \\
1810-1814\end{array}$ \\
\hline Bedford (1) & $\begin{array}{l}\text { J. Hertel de } \\
\text { Rouville }\end{array}$ & N. Coffin & J. Steele & W. Moore & $\ldots \ldots \ldots \ldots \cdots \cdots \cdots, \ldots$ & John Jones & A. Desbleds \\
\hline $\begin{array}{l}\text { Buckinghamshire } \\
\text { (2) }\end{array}$ & $\begin{array}{l}\text { J. Duchesnay } \\
\text { J. de Tonnacour }\end{array}$ & $\begin{array}{l}\text { J. Craigie } \\
\text { G. Allsopp }\end{array}$ & L. Gouin & $\begin{array}{l}\text { L. Proulx } \\
\text { F. LeGendre }\end{array}$ & J.-B. Hébert & 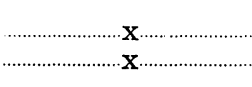 & $\ldots \cdots \cdots \cdots \cdots \cdot \mathbf{X}$ \\
\hline Cornwallis (2) & $\begin{array}{l}\text { J. Digé } \\
\text { P.-L. Panet }\end{array}$ & $\begin{array}{l}\text { P. Sirois } \\
\text { 1797: P. Taché * } \\
\text { A. Menut }\end{array}$ & J. Boucher & $\begin{array}{l}\text { J.-N. Perrault } \\
\text { A. Roi }\end{array}$ & $\begin{array}{l}\text { J.-L. Borgia } \ldots \\
\text { J. Robitaille }\end{array}$ & $\mathbf{X}$ & $x$ \\
\hline Devon (2) & $\begin{array}{l}\text { F. Dambourges } \\
\text { J. Tod }\end{array}$ & $\begin{array}{l}\text { N. Dorion } \\
\text { F. Bernier.. }\end{array}$ & J.-B. Pelletier & J.-B. Fortin & $\ldots$ & $(\cdots \cdots \cdots \cdots \cdots \cdots \cdots$ & 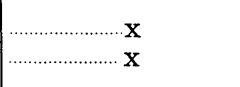 \\
\hline Dorchester (2) & $\begin{array}{l}\text { G. Taschereau } \\
\text { L. de Salaberry }\end{array}$ & $\begin{array}{l}\text { C. Bégin } \\
\text { A. Dumas }\end{array}$ & 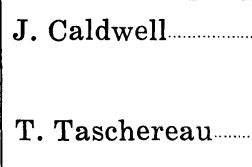 & $\ldots \ldots \ldots \ldots \ldots \ldots$ & P. Langlois & $\begin{array}{r}\text { T. Taschereau } \\
\\
.\end{array}$ & $\begin{array}{l}\text { J. Caldwell } \\
\frac{1813:}{\mathrm{T} . \text { Taschereau }} \\
\ldots \ldots \ldots \ldots \ldots \\
\mathrm{x}\end{array}$ \\
\hline Effingham (2) & $\begin{array}{l}\text { J. Jordan } \\
\text { J. Lacroix }\end{array}$ & 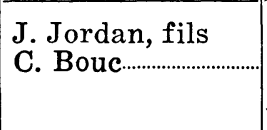 & $\begin{array}{l}\text { A. Nadon } \\
\text { 1803: A. Shaw } \\
\end{array}$ & T. Porteous & 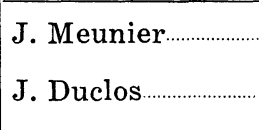 & 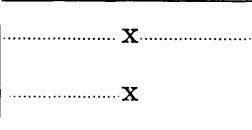 & J. Malboeuf \\
\hline Gaspé (1) & E. O'Hara & $\ldots \ldots \ldots$ & W. Vondenvelden & G. Pyke & 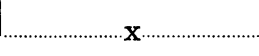 & 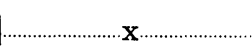 & 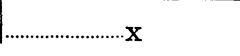 \\
\hline
\end{tabular}

* Les noms soulignés précédés d'une date sont ceux des députés élus lors des élections complémentaires. 
LES DÉPUTÉS À L'ASSEMBLÉE LÉGISLATIVE DU BAS-CANADA, 1792-1814 (suite)

\begin{tabular}{|c|c|c|c|c|c|c|c|}
\hline CIRCONSCRIPTION & 1er PARLEMENT & 2e PARLEMENT & 3e PARLEMENT & 4e PARLEMENT & 5e PARLEMENT & 6e PARLEMENT & 7e PARLEMENT \\
\hline Hampshire (2) & $\begin{array}{l}\text { J. Boudreau } \\
\text { M. McNider }\end{array}$ & $\begin{array}{l}\text { J. Planté } \\
\text { F. Huot }\end{array}$ & $\mathbf{x}$ & J. Duchesnay & F. Huot & $\mathbf{X}$ & F. Larue \\
\hline Hertford (2) & $\begin{array}{l}\text { L. Dunière } \\
\text { P. Marcoux }\end{array}$ & F. Têtu & $\begin{array}{l}\text { M. Tellier } \\
\text { L. Blais }\end{array}$ & $\begin{array}{l}\text { L. Turgeon } \\
\text { E.-F. Roy }\end{array}$ & $\mathbf{X}$ & F. Blanchet & 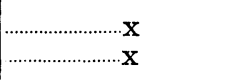 \\
\hline Huntingdon (2) & $\begin{array}{l}\text { G.-H. Dupré } \\
\text { G. de Lorimier }\end{array}$ & $\begin{array}{l}\text { J. Perineault } \\
\text { J. Perrault }\end{array}$ & J.-B. Raymond & A. Mackenzie & $\begin{array}{l}\text { L. de Salaberry } \\
\text { J.-A. Panet }\end{array}$ & S. Sewell & E. Henry \\
\hline Kent (2) & $\begin{array}{l}\text { R. Boileau } \\
\text { P. Legras- } \\
\text { Pierreville }\end{array}$ & $\begin{array}{l}\text { Jacques Viger } \\
1798: \text { Berthelot } \\
\text { D'Artigny } \\
\text { A. Menard } \\
\text { Lafontaine }\end{array}$ & . & P. Weilbrenner & $\begin{array}{l}\text { J. Planté } \\
\text { L.-J. Papineau... }\end{array}$ & P.-D. Debartzch & $-\cdots \cdots \cdots \cdots \cdot \mathbf{X}$ \\
\hline Leinster (2) & $\begin{array}{l}\text { F.-A. Laroque } \\
\frac{1794:}{\text { G. Macbeath }} \\
\text { B. Panet }\end{array}$ & Joseph Viger & $\begin{array}{l}\text { J.Beaumont } \\
\text { J. Archambault.... }\end{array}$ & C. de Lanaudière & $\begin{array}{l}\text { J. Faribault } \\
\text { J. Turgeon }\end{array}$ & $\begin{array}{l}\text { T. Taschereau } \\
\text { B. Panet }\end{array}$ & $\begin{array}{l}\text { J. Archambault } \\
\text { D.-B. Viger }\end{array}$ \\
\hline Montréal-Est (2) & $\begin{array}{l}\text { J. Frobisher } \\
\text { J. Richardson }\end{array}$ & $\begin{array}{l}\text { A. Auldjo } \\
\text { L.-C. Foucher }\end{array}$ & $\begin{array}{l}\text { P.-L. Panet } \\
\text { F. Badgely }\end{array}$ & $\begin{array}{l}\text { J. Richardson } \\
\text { J.-M. Mondelet .... }\end{array}$ & J. Stuart & J. Papineau & S. Sewell \\
\hline $\begin{array}{c}\text { Montréal-Ouest } \\
\text { (2) }\end{array}$ & $\begin{array}{l}\text { J.-B. Durocher } \\
\text { J. McGill }\end{array}$ & $\begin{array}{l}\text { J. Papineau } \\
\text { D. Viger }\end{array}$ & $\begin{array}{l}\text { J. Perinault } \\
\text { J. McGill }\end{array}$ & L. Chabouillez & $\begin{array}{l}\text { W. McGillivray } \\
\text { D.-B. Viger }\end{array}$ & T. McCord & $\begin{array}{l}\text { A. McLeod } \\
\text { E.-N. St-Dizier }\end{array}$ \\
\hline
\end{tabular}

* Les noms soulignés précédés d'une date sont ceux des députés élus lors des élections complémentaires. 
LES DÉPUTÉS À L’ASSEMBLÉE LÉGISLATIVE DU BAS-CANADA, 1792-1814 (suite)

\begin{tabular}{|c|c|c|c|c|c|c|c|}
\hline CIRCONSCRIPTION & 1er PARLEMENT & 2e PARLEMENT & 3e PARLEMENT & 4e PARLEMENT & 5e PARLEMENT & 6e PARLEMENT & 7e PARLEMENT \\
\hline Montréal (2) & $\begin{array}{l}\text { J. Papineau } \\
\text { J. Walker }\end{array}$ & $\begin{array}{l}\text { J.-M. Ducharme } \\
\text { E. Guy }\end{array}$ & $\begin{array}{l}\text { J. Papineau } \\
\text { T. Walker }\end{array}$ & $\begin{array}{l}\text { B. J. Frobisher } \\
\text { L. Roi dit } \\
\text { Portelance }\end{array}$ & $\begin{array}{l}\text { J.-B. Durocher } \\
\text {. }\end{array}$ & $\ldots \cdots \cdots \cdots$ & $\frac{1811: \text { J. Stuart }}{\ldots}$ \\
\hline$\underset{(2)}{\text { Northumberland }}$ & $\begin{array}{l}\text { P. Bédard } \\
\text { J. Dufo... }\end{array}$ & J. Fisher & J.-M. Poulin & $\mathbf{x}$ & A. Caron & 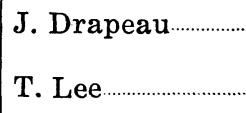 & $\frac{1811: \text { A. Caron }}{\mathrm{x}}$ \\
\hline Orléans (1) & N. Boisseau & J. Martineau & $\ldots \ldots \ldots \ldots \ldots \ldots$ & 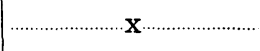 & 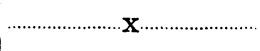 & $\ldots \ldots . . . \times x$ & L. Blouin \\
\hline $\begin{array}{l}\text { Québec, basse- } \\
\text { ville (2) }\end{array}$ & $\begin{array}{l}\text { R. Lester } \\
\text { J. Young.. }\end{array}$ & A. Raby & R. Lester & L. de Salaberry & $\begin{array}{l}\text { P. Bédard } \\
\text { James Jones }\end{array}$ & 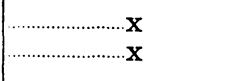 & $\begin{array}{l}\text { P. Bruneau } \\
\text { J. Mure }\end{array}$ \\
\hline $\begin{array}{l}\text { Québec, haute- } \\
\text { ville (2) }\end{array}$ & 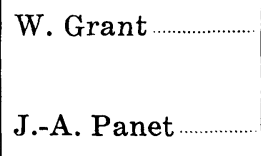 & $\mathbf{x}$ & $\mathbf{x}$ & 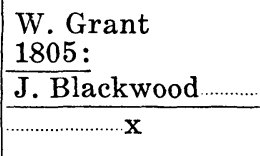 & C. Dénéchaud & $\ldots \ldots \ldots \ldots \ldots$ & J. Irvine \\
\hline Québec (2) & $\begin{array}{l}\text { L. de Salaberry } \\
\text { 1793: Berthelot } \\
\text { D'Artigny } \\
\text { D. Lynd }\end{array}$ & $\begin{array}{l}\text { J. Black } \\
\text { L. Paquet }\end{array}$ & $\begin{array}{l}\text { Berthelot } \\
\text { D'Artigny } \ldots \ldots . . . \\
\qquad . . .\end{array}$ & $\begin{array}{l}\text { P.-A. DeBonne } \\
\text { P............. }\end{array}$ & R. Gray $\ldots \ldots \ldots \ldots \ldots \ldots$ & $\quad x \ldots \ldots \ldots$ & $\begin{array}{l}\text { L. Gauvreau } \\
\text { J.-B. Bédard }\end{array}$ \\
\hline Richelieu (2) & $\begin{array}{l}\text { B.-H. Cherrier } \\
\text { P. Guerout }\end{array}$ & C. Millette & $\begin{array}{l}\text { L. Hubert } \\
\text { C. Benoit dit } \\
\text { Livernois }\end{array}$ & $\begin{array}{l}\text { L. Bourdages } \\
\text { L. Brodeur. }\end{array}$ & H. Delorme & 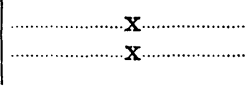 & $\ldots+\cdots \cdots \cdots+\cdots \cdot \ldots$ \\
\hline
\end{tabular}

* Les noms soulignés précédés d'une date sont ceux des députés élus lors des élections complémentaires. 
LES DÉPUTÉS À L’ASSEMBLÉE LÉGISLATIVE DU BAS-CANADA, 1792-1814 (suite et fin)

\begin{tabular}{|c|c|c|c|c|c|c|c|}
\hline CIRCONSCRIPTION & 1er PARLEMENT & 2e PARLEMENT & 3e PARLEMENT & 4e PARLEMENT & 5e PARLEMENT & 6e PARLEMENT & 7e PARLEMENT \\
\hline $\begin{array}{c}\text { Saint-Maurice } \\
(2)\end{array}$ & $\begin{array}{l}\text { T. Coffin } . . . \\
\text { A. Rivard }\end{array}$ & N. Montour & M. Bell & $\begin{array}{l}\text { D. Munro } \\
\text { M. Caron }\end{array}$ & T. Coffin & L. Gugy & F. Caron \\
\hline Surrey (2) & $\begin{array}{l}\text { F. Mailhot } \\
\text { P. de Rocheblave }\end{array}$ & O. Durocher & $\begin{array}{l}\text { F. Lévêsque } \\
\text { 1803: A. Caron } \\
\end{array}$ & $\begin{array}{l}\text { J. Cartier } \\
\text { N. de Rocheblave } \\
\text { 1806: P. Lussier }\end{array}$ & P. Chagnon & $\begin{array}{l}\text { P. Bédard } \\
\text { J. Beauchamp }\end{array}$ & J. Bédard \\
\hline $\begin{array}{c}\text { Trois-Rivières } \\
\text { (2) }\end{array}$ & $\begin{array}{l}\text { J. Lees } \\
\text { N. St-Martin }\end{array}$ & P.-A. DeBonne & $=\mathrm{X}$ & $\frac{1807: \text { E. Hart }}{\text { L.-C. Foucher }}$ & J. Badeaux & M. Bell $\ldots \ldots \ldots$ & T. Coffin \\
\hline Warwick (2) & $\begin{array}{l}\text { P. de Lavalterie } \\
\text { L. Olivier }\end{array}$ & $\begin{array}{l}\text { G. de Lanaudière } \\
\text { J. Cuthbert }\end{array}$ & R. Cuthbert & 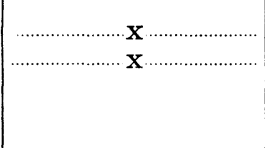 & $\begin{array}{r}\mathbf{X} \\
\mathbf{X}\end{array}$ & $\ldots \cdots \cdots \cdots \cdots \cdots+\cdots$ & $\begin{array}{l}\text { L. Olivier } \\
\text { 1812: } \\
\text { R. Cuthbert } \\
\end{array}$ \\
\hline$\underset{\text { (1) }}{\text { William Henry }}$ & J. Barnes & J. Sewell & 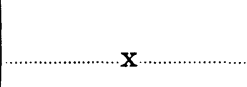 & $\mathbf{x}$ & $\mathbf{x}$ & E. Bowen & $\ldots . . \times$ \\
\hline York (2) & $\begin{array}{l}\text { P.-A. DeBonne } \\
\text { C. de Lotbinière }\end{array}$ & $\begin{array}{l}\text { J. Lacroix } \\
\text { J. Ethier }\end{array}$ & $\begin{array}{l}\text { J. Bédard } \\
\text { L.-C. Foucher }\end{array}$ & $\begin{array}{l}\text { E. Dumont } \\
\text { J. Mure }\end{array}$ & $\begin{array}{l}\text { J. Trestler } \\
\text { X }\end{array}$ & P. St-Julien & F. Bellet \\
\hline
\end{tabular}

* Les noms soulignés précédés d'une date sont ceux des députés élus lors des élections complémentaires. 